\title{
Effects of Potassium Deficiency on Growth of Pakchoi in Cold and Dry Areas
}

\section{Shi-Heng LI ( $\nabla$ 18847163576@163.com )}

Inner Mongolia Agricultural University

\section{Wei-Ming LI}

Qingdao Zibainong

Ling-Hao BU

Inner Mongolia Agricultural University

\section{Original Article}

Keywords: potash fertilizer, cabbage, nutrient solution, substrate cultivation

Posted Date: February 23rd, 2021

DOI: https://doi.org/10.21203/rs.3.rs-229701/v1

License: (c) (i) This work is licensed under a Creative Commons Attribution 4.0 International License. Read Full License 


\section{Abstract}

[Background] To reveal the effect of potassium deficiency on the growth and development of pakchoi in cold and dry areas.

[Results] The effect of potassium deficiency on the growth status of P. pakchoi in cold and dry areas was significant. P. pakchoi treated with potassium-free nutrient solution had the best growth, the largest number of leaves, leaf length, leaf width, leaf area, root length and weight; P. pakchoi treated with $100 \%$ potassium deficiency had the smallest plant, leaf length, leaf width, leaf area, root length and weight; P. pakchoi treated with potassium deficiency $50 \%$ had the middle growth and development status, and the root ratio was the smallest in $\mathrm{d} 21$. [Conclusion] Potassium can effectively promote the growth and development of pakchoi in cold and dry areas, increase its root length, leaf width, leaf area, weight, etc. The quality of the upper part and the underground part of d,21 of pakchoi treated with deficiency is the most different.

\section{Background}

Inner Mongolia is a middle temperate monsoon climate area with less and uneven precipitation, shortage of water resources and low temperature in the whole year, which makes crop growth need certain environmental conditions. This experiment shows the effect of potassium deficiency on the growth and development of pakchoi in cold and dry areas. To guide the cultivation and growth model of pakchoi in Inner Mongolia combined with fertilization management to improve the yield of pakchoi ${ }^{[1-3]}$ With the improvement of people's living standard, people pay more attention to the pursuit of health.As a kind of green organic vegetable, pakchoi is rich in many nutrients needed by human body, and gradually becomes an important choice for people to eat. Potassium is one of the three elements of fertilizer, which is involved in almost all biochemical processes in plant growth and development. It is called quality element and stress resistance element ${ }^{[4]}$ The results showed that the leaf senescence of pakchoi was accelerated under the condition of potassium deficiency. The results showed that with the increase of potassium concentration in nutrient solution, the fresh quality, cell yield, leaf length, plant height, leaf number, dry quality and root dry quality of each plant increased to varying degrees. Soilless cultivation technique was applied in this study ${ }^{[5-6]}$ The effects of potassium deficiency on leaf number, root length, leaf length, leaf width, underground fresh weight, aboveground fresh weight, underground dry weight, aboveground dry weight, aboveground dry weight, leaf area, root-crown ratio in cold and dry areas of Inner Mongolia were studied.

\section{Materials And Methods 1.1 Test materials}

In this study, pakchoi was used as experimental material, and the nutrient solution was green leafy vegetable nutrient solution ${ }^{[7]}$ and set up no potassium deficiency (CK), potassium deficiency $50 \%$ and 


\subsection{Test methods}

1.2.1 sowing and planting of pakchoi from May to August 2018, In Inner Mongolia Agricultural University practice teaching base farm greenhouse; Using a hole plate, The seedling plate is 128 points, Sowing on 31 May 2020, One hole and one grain; It was planted in greenhouse cultivation trough on June 22. Test design 3 treatments, Potassium concentrations in nutrient solution were $250 \mathrm{mg} / \mathrm{L}(\mathrm{CK}), 125 \mathrm{mg} / \mathrm{L}$ and 0 , Each group was tested in different cultivation tanks, 22 plants per slot, $25 \mathrm{~cm}$. plant spacing Water the nutrient solution every 7 days, $50 \mathrm{ml}$. per plant When sampling, Three random plants per week, For five weeks, And the corresponding indicators are measured.

Table 1 Nutrients for green leafy vegetables

\begin{tabular}{|llll|}
\hline Fertilizer name & Amount/(mg/L) & & \\
\cline { 2 - 4 } & $\begin{array}{l}\text { No shortage of K (K } \\
\text { C) }\end{array}$ & $\begin{array}{l}\text { K50\% of } \\
\text { vacancies }\end{array}$ & $\begin{array}{l}\text { K100\% of } \\
\text { vacancies }\end{array}$ \\
\hline Calcium nitrate & 1260 & 1260 & 1260 \\
\hline Potassium sulfate & 250 & 125 & 0 \\
\hline $\begin{array}{l}\text { Dihydrogen phosphate } \\
\text { ammonia }\end{array}$ & 350 & 350 & 350 \\
\hline Magnesium sulfate & 537 & 537 & 537 \\
\hline Ammonium sulfate & 237 & 237 & 237 \\
\hline
\end{tabular}

The leaf length, leaf width and root length of pakchoi were measured directly by millimeter, and the leaf area of each pakchoi was calculated equal to the average leaf length $x$ the average leaf width of the maximum leaf length, and the number of leaves was calculated. The fresh weight of the upper and underground parts of rapeseed was measured by $1 / 10000$ days, then dried by dryer $\left(78^{\circ} \mathrm{C}\right)$ to constant weight, and the dry weight of the above ground and underground parts was measured by $1 / 10000$ days.

WPS office Excel,SPSS 18.0 is the data processing software used

Fresh weight of root to crown ratio $=$ ground/ground fresh weight (1)

Water content $/ \%=($ fresh weight-dry weight) $/$ fresh weight $\times 100(2)$

\section{Fruit And Analysis}




\subsection{Effects of potassium deficiency on leaf count of pakchoi}

Figure 1 shows that, Under the same nutrient solution, The leaf number of pakchoi increased with the extension of planting time from day 14 , The effect of potassium deficiency on leaf number of pakchoi was significant. At the same time, As potassium deficiency deepens, The number of pakchoi leaves showed a downward trend. After treatment of 7,14,21,28,35 $d$, The results showed that the leaf number of pakchoi was the smallest under $100 \%$ potassium deficiency, $6,6,12,13,17$, The number of leaves of pakchoi treated with potassium deficiency $50 \%$ nutrient solution was higher than that treated with potassium deficiency $100 \%$ nutrient solution, $7,7,12,15,18$, The maximum number of leaves of pakchoi was $8,8,13,17,22$, It was significantly higher than the number of leaves treated with potassium deficiency $50 \%$ and potassium deficiency $100 \%$ nutrient solution, The results showed that potassium could promote the increase of leaf number.

\subsection{Effects of potassium deficiency on leaf length, leaf width and leaf area of pakchoi}

Figure 2 shows that, Under the same nutrient solution, The length of pakchoi leaves increased with the increase of planting time, The effect of potassium deficiency on leaf length of pakchoi was significant. At the same time, As potassium deficiency deepens, The length of pakchoi leaves showed a downward trend. After treatment of 7,14,21,28,35 d, The results showed that the leaf length of pakchoi was the smallest under $100 \%$ potassium deficiency, 3.3,7.87,15.93,20.93,24.77 cm, respectively The length of leaf of cabbage treated with potassium deficiency $50 \%$ nutrient solution was higher than that treated with potassium deficiency $100 \%$ nutrient solution, $3.67,8.5,17.7,22.47,25 \mathrm{~cm}$, respectively After treatment with potassium-free nutrient solution, the maximum length of leaves was 4.9,10.7,19.2,23.5,26.2 cm, respectively The length of leaf length of pakchoi treated with potassium deficiency $50 \%$ and potassium deficiency $100 \%$ nutrient solution, The results showed that potassium could promote the growth of leaf length.

Figure 3 shows that, Under the same nutrient solution, The width of pakchoi leaves increased with the extension of planting time, The effect of potassium deficiency on leaf width of pakchoi was significant. At the same time, As potassium deficiency deepens, The width of pakchoi leaves showed a downward trend. After treatment of 7,14,21,28,35d, The results showed that the leaf width of pakchoi was the smallest under $100 \%$ potassium deficiency, $1.9,2.7,6.97,8.5$ and $9.83 \mathrm{~cm}$, respectively The width of leaf width of cabbage treated with potassium deficiency $50 \%$ nutrient solution was higher than that of cabbage treated with potassium deficiency $100 \%$ nutrient solution, $2.2,3.87,7.87,9.4,10.23 \mathrm{~cm}$, respectively After treatment with potassium-free nutrient solution, the maximum width of leaf width was 3.27,4.87,8.1,10.57 and $11.7 \mathrm{~cm}$, respectively The leaf width of pakchoi treated with potassium deficiency 
$50 \%$ and potassium deficiency $100 \%$ nutrient solution was significantly higher, The results showed that potassium could promote the growth of leaf width of pakchoi.

Fig .4 shows that the leaf area of pakchoi increased with the prolongation of planting time under the same nutrient solution treatment, and the effect of potassium deficiency treatment on leaf area of pakchoi was significant. In the same period, with the deepening of potassium deficiency, the leaf area of pakchoi decreased. When treated with 7,14,21,28 and $35 d$, the results showed that the leaf area of pakchoi was the smallest under $100 \%$ potassium deficiency $, 6.27,21.249,111.032,177.905,243.49 \mathrm{~cm}^{2}$, and the leaf area under $50 \%$ potassium deficiency was higher than that under $100 \%$ potassium deficiency $, 8.07,32.895,139.299,211.218,255.1$ The leaf area of pakchoi was $16.023,52.109,155.52,248.395$ and $306.54 \mathrm{~cm}^{2}$, which was significantly higher than that of pakchoi treated with potassium deficiency $50 \%$ and potassium deficiency $100 \%$ nutrient solution, indicating that potassium element could significantly promote the increase of pakchoi leaf area.

\subsection{Effects of potassium deficiency on root length of cabbage}

Figure 5 shows that, Under the same nutrient solution, The root length of pakchoi increased with the increase of planting time, The effect of potassium deficiency on root length of pakchoi was significant. At the same time, As potassium deficiency deepens, The root length of pakchoi decreased. After treatment of $7,14,21,28,35 d$, The results showed that the root length of pakchoi was the smallest under $100 \%$ potassium deficiency, 4,6.3,9.8,10.4 and $15.8 \mathrm{~cm}$, respectively The root length of pakchoi treated with potassium deficiency $50 \%$ nutrient solution was higher than that treated with potassium deficiency $100 \%$ nutrient solution, 4.73,6.3,10.7,13.8,16.4 cm, respectively After treatment with potassium-free nutrient solution, the root length of cabbage was $6.37,6.97,11.87,14.13$ and $17.9 \mathrm{~cm}$, respectively It was significantly higher than the root length of cabbage treated with potassium deficiency $50 \%$ and potassium deficiency $100 \%$ nutrient solution, The results showed that potassium could promote the growth of root length of cabbage.

\subsection{Effects of potassium deficiency on fresh weight, dry weight and root-crown ratio of pakchoi}

Figure 6 shows that, Under the same nutrient solution, The fresh weight of pakchoi increased with the extension of planting time, Effects of potassium deficiency treatment on fresh weight of pakchoi were not significant in 7,14 d, significant differences d 21,28,35. At the same time, As potassium deficiency deepens, The fresh weight of the upper part of the cabbage field showed a downward trend. After treatment of 7,14,21,28,35 d, The results showed that the fresh weight of pakchoi was the smallest under $100 \%$ potassium deficiency, $1.68,2.88,16.36,53.66,115.56 \mathrm{~g}$, respectively The fresh weight of the upper part of the cabbage was higher than that of the potassium deficiency $100 \%$ nutrient solution, 
$1.72,3.11,17.9,63.42,137.19 \mathrm{~g}$, respectively After treatment with potassium-free nutrient solution, the maximum fresh weight of the upper part of the vegetable field was $1.76,5.57,27,110.5,237.87 \mathrm{~g}$, respectively It is obviously higher than the fresh weight of the upper part of the vegetable field treated with potassium deficiency $50 \%$ and potassium deficiency $100 \%$ nutrient solution, The results showed that potassium could promote the increase of fresh weight in the upper part of cabbage, most obvious in $d 35$.

Figure 7 shows that, Under the same nutrient solution, The dry weight of the upper part of the cabbage field increased with the extension of planting time, Effects of potassium deficiency treatment on dry weight of the upper part of cabbage were not significant $d 7,14$, significant differences $d 21,28,35$. At the same time, As potassium deficiency deepens, The dry weight of the upper part of the cabbage field is decreasing. After treatment of $7,14,21,28,35 d$, The results showed that the dry weight on the ground of pakchoi was the smallest under $100 \%$ potassium deficiency, $0.173,0.257,1.03,2.1,7.087 \mathrm{~g}$,, respectively The dry weight of the upper part of the cabbage was higher than that of the potassium deficiency $100 \%$ nutrient solution, $0.18,0.493,1.93,4,10.683 \mathrm{~g}$, respectively After treatment with potassium-free nutrient solution, the maximum dry weight of the upper part of the vegetable field was $0.237,0.557,2.63,4.03$ and $11.13 \mathrm{~g}$, respectively It was significantly higher than the dry weight of the upper part of the vegetable field treated with potassium deficiency $50 \%$ and potassium deficiency $100 \%$ nutrient solution, The results showed that potassium could promote the increase of dry weight in the upper part of cabbage, most obvious in $\mathrm{d} 35$.

Figure 8 shows that under the same nutrient solution treatment, the root and crown ratio of pakchoi did not change obviously with the prolongation of planting time, and the effect of potassium deficiency treatment on root and crown ratio of pakchoi was significant. The root-to-crown ratio of pakchoi increased first and then decreased $d 7,14,21,28$ and 35 treated with potassium deficiency $50 \%$ and $100 \%$ nutrient solution, and reached the minimum value in $\mathrm{d} 21$, indicating that the quality difference between the upper part and the underground part of pakchoi treated with d,21 was the greatest.

\subsection{Effects of potassium deficiency on water content of cabbage}

Figure 9 shows that, Under different nutrient solutions, The water content of pakchoi was above $88 \%$ with the extension of planting time, At d 28, the water content reaches its maximum, No potassium deficiency, potassium deficiency $50 \%$, potassium deficiency $100 \%$ nutrient solution irrigated. The water content of pakchoi was $96.3 \%, 95 \%, 96.8 \%$, The water content of cabbage was higher $d 7,14$ and 21 . The possible reason is that the initial stage of colonization is to adapt to the new environment, the water content in the matrix is insufficient, the water supply is insufficient, and the water content of pakchoi increases as a whole with watering and replenishing the root system of pakchoi. 


\section{Results}

The effect of potassium deficiency on the growth status of P. pakchoi in cold and dry areas was significant. P. pakchoi treated with potassium-free nutrient solution had the best growth, the largest number of leaves, leaf length, leaf width, leaf area, root length and weight; P. pakchoi treated with $100 \%$ potassium deficiency had the smallest plant, leaf length, leaf width, leaf area, root length and weight; P. pakchoi treated with potassium deficiency $50 \%$ had the middle growth and development status, and the root ratio was the smallest in $\mathrm{d} 21$.

\section{Discussion And Conclusions}

In this study, the effects of potassium deficiency on the growth of pakchoi were studied. The results showed that the growth of pakchoi was severely inhibited by potassium deficiency, such as weak growth, few leaves, yellow leaves, high morbidity and mortality, long leaves, short leaves, small leaf area, fresh weight on the ground and small dry weight on the ground ${ }^{[8-12]}$ This is Sun Ting ${ }^{[13]}$ The effects of different potassium fertilizer on the growth, nutrient absorption and soil nutrient content of pakchoi in greenhouse were similar. The results showed that adding $20 \%$ potassium fertilizer on the basis of the main formula could improve the soil condition, improve the quality and adaptability of pakchoi.

The results showed that the root-crown ratio of pakchoi was the smallest in $\mathrm{d} 21$, and the difference between fresh weight and dry weight was the largest in $d 35$. At the $21 \mathrm{st} d$, the quality difference between the upper part of cabbage and the underground part was the biggest, and the effective complement of potassium fertilizer could effectively alleviate the inhibition of potassium deficiency on the growth of cabbage. The effect of potassium application on the root-to-crown ratio and soluble sugar of pakchoi could be further studied ${ }^{[14-17]}[$

\section{Declarations}

Ethics approval and consent to participate All authors agree to publish All test data are true and reliable Articles not published, no conflict of interest Funding $\triangle T$ The cost of publishing is borne by the author Authors' contributions $₫$ All authors work together to design, collect and analyze data.

Acknowledgements:Thank all the authors for their hard work Authors' informationखLi Shiheng (1996-), male, master's degree, research direction is horticultural plant physiology and ecology. Tel『 13733312721,Email囚18847163576@163.com

\section{References}

1. Zou Fuzhen. Effects of inorganic-organic mixed improvers on remediation of acidic polymetallic contaminated soils [D].]; and Guangzhou: South China Agricultural University

2. Zhao Hongwei S, Lichao J, Conghui YY, Jinxu Fu, Jiapeng Z, English M, Meirong Xu, Yongxu L, Wang Hao. Effects of Nitrogen, Phosphorus and Potassium Fertilizer Application on Growth and Yield of 
Japonica Rice in Cold Land [J].]1 Journal of Northeast Agricultural University,2020,51(12):1-13

3. Miao Qi D, Yuanyuan J, Jie et al. Effect of potassium supply level on assimilate and potassium distribution of pakchoi [J]. North Horticulture,2017(16):121-127

4. Yan Xiaohai. Nutritional and Health Care Role of Potassium J]. Oriental Dietotherapy and Health Care,2007(8): $26+43$

5. Wang Fengxing. Integrated Soilless Cultivation Technology for Organic Vegetable Water \& Fertilizer [J].] Agriculture and Technology,2019,39(4):18-19

6. Jiang Weijie Z, Guanghua W, Hao et al. Organic ecotype soilless cultivation technique and its nutritional physiological basis [Journal of J], Horticulture,1996,23(2):43-45

7. Wen Qingqin. Effects of Different Concentrations of Nutrients on the Growth of J]. Vegetable Yangtze River Vegetables,2009(8):55-56

8. Bingsong Z, Xiaojian C, Jiang Dean, et al. The effect of potassium on photosynthetic rate, Rubisco and RCA of plants [J].]; and Journal of Zhejiang Agriculture and Forestry University 19(1):104-108

9. Lu Zhifeng Lu, Jianwei P, Yonghui et al. Physiological mechanism of potassium regulating plant photosynthesis [J].]; and Journal of Plant Physiology,2016,52(12):1773-1784

10. Rao Lihua X, Jianming J Dean, et al. Effects of Potassium Nutrition on Photosynthesis and Yield Formation of Tomato J]. Journal of Zhejiang Agricultural University,1989(04):9-16

11. Pan Yonghui. Effects of potassium deficiency stress on leaf area formation and photosynthetic characteristics of vegetable oil-selling population Wuhan: Huazhong Agricultural University

12. Tian Yueyue. Effects of long-term potassium deficiency on root exudates and rhizosphere microecology of facility tomato D]. Shenyang [Shenyang Agricultural University]

13. Sun Ting L Xiaoli, Wang Menglan, et al. Effects of different potassium fertilizer dosage on growth, nutrient absorption and soil nutrient content of pakchoi in greenhouse [J]. Jiangsu Agricultural Science,2020,48(12):134-137

14. Han Y. Effects of potassium application on peanut growth, yield and quality [D].] under UV-B enhancement Nanjing: Nanjing University of Information Engineering

15. Shen Changwei. Effects of potassium application on physiological and molecular mechanisms of sugar synthesis and distribution in pear leaves and D]. fruit Nanjing: Nanjing Agricultural University

16. Wu X. Effect of potassium application on yield and quality of Huangguan pear and potassium nutrition [D].].1 Nanjing: Nanjing Agricultural University

17. Wang L. Effect of potassium application on yield quality and potassium absorption and utilization of drip irrigation potato under D]. film Hohhot: inner Mongolia Agricultural University

\section{Figures}




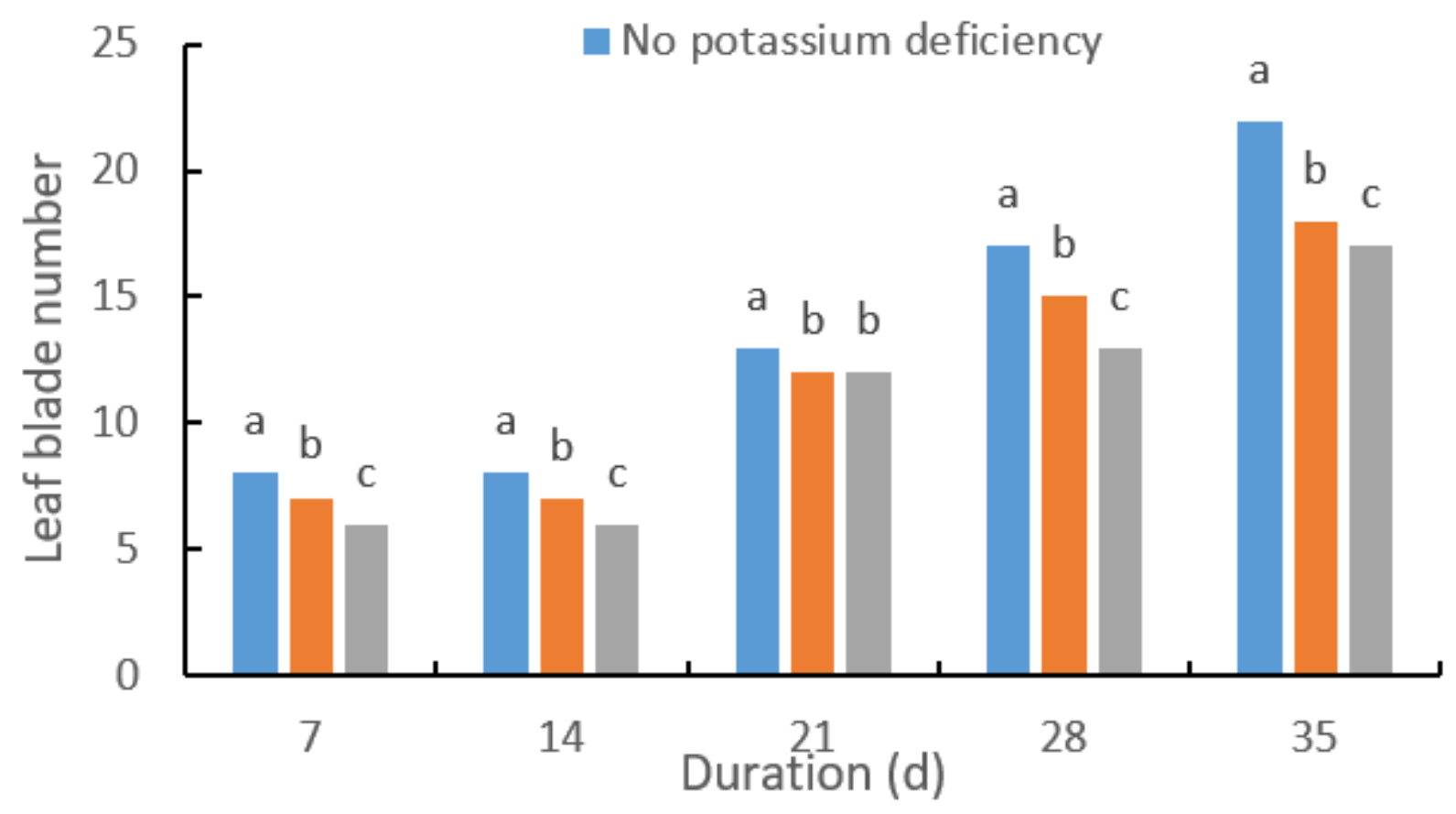

Figure 1

Effect of different potassium treatment on leaf number of pakchoi

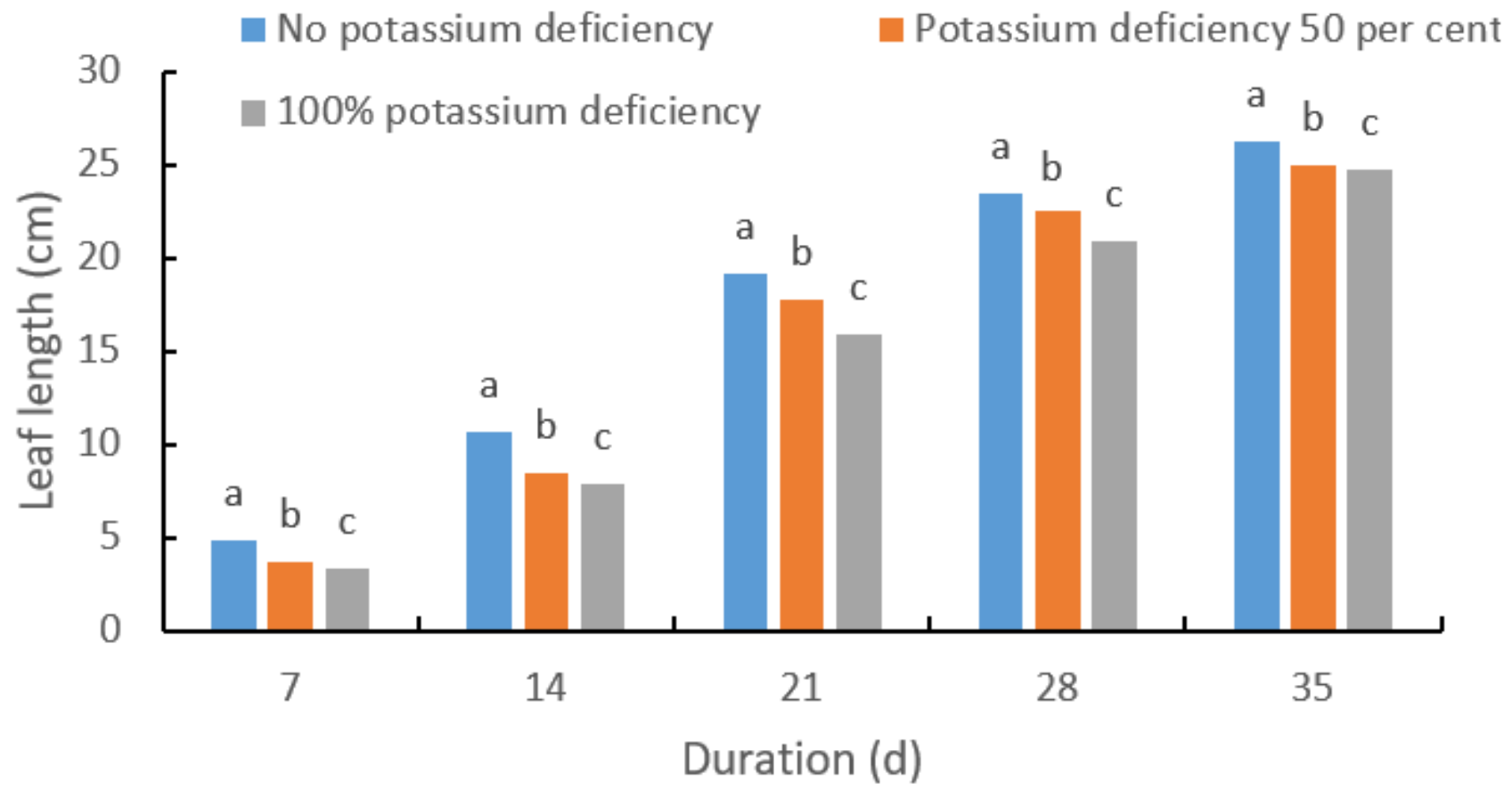


Figure 2

Effects of different potassium treatments on leaf length of cabbage

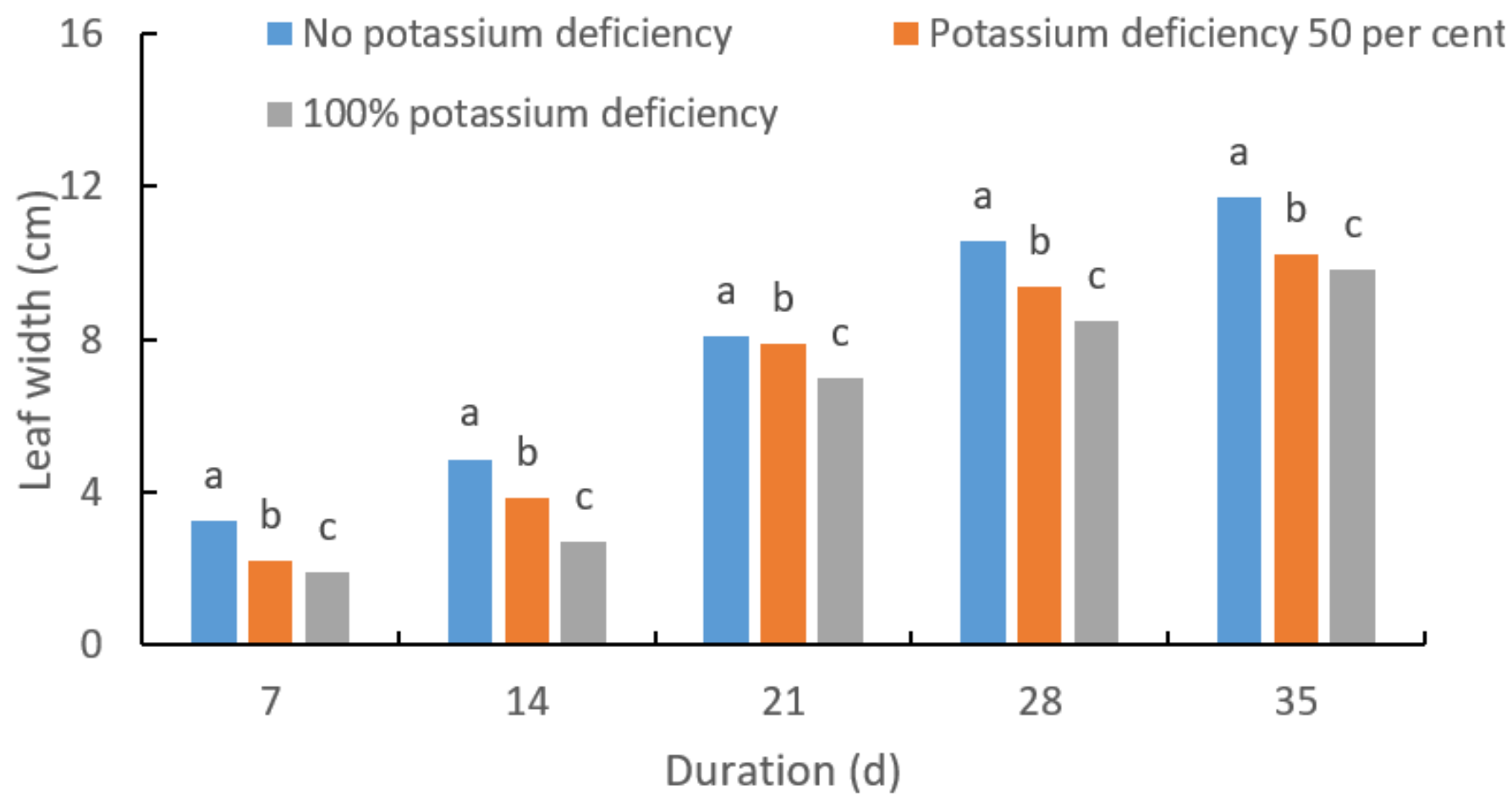

Figure 3

Effects of different potassium treatments on leaf width of Oryza sativa 


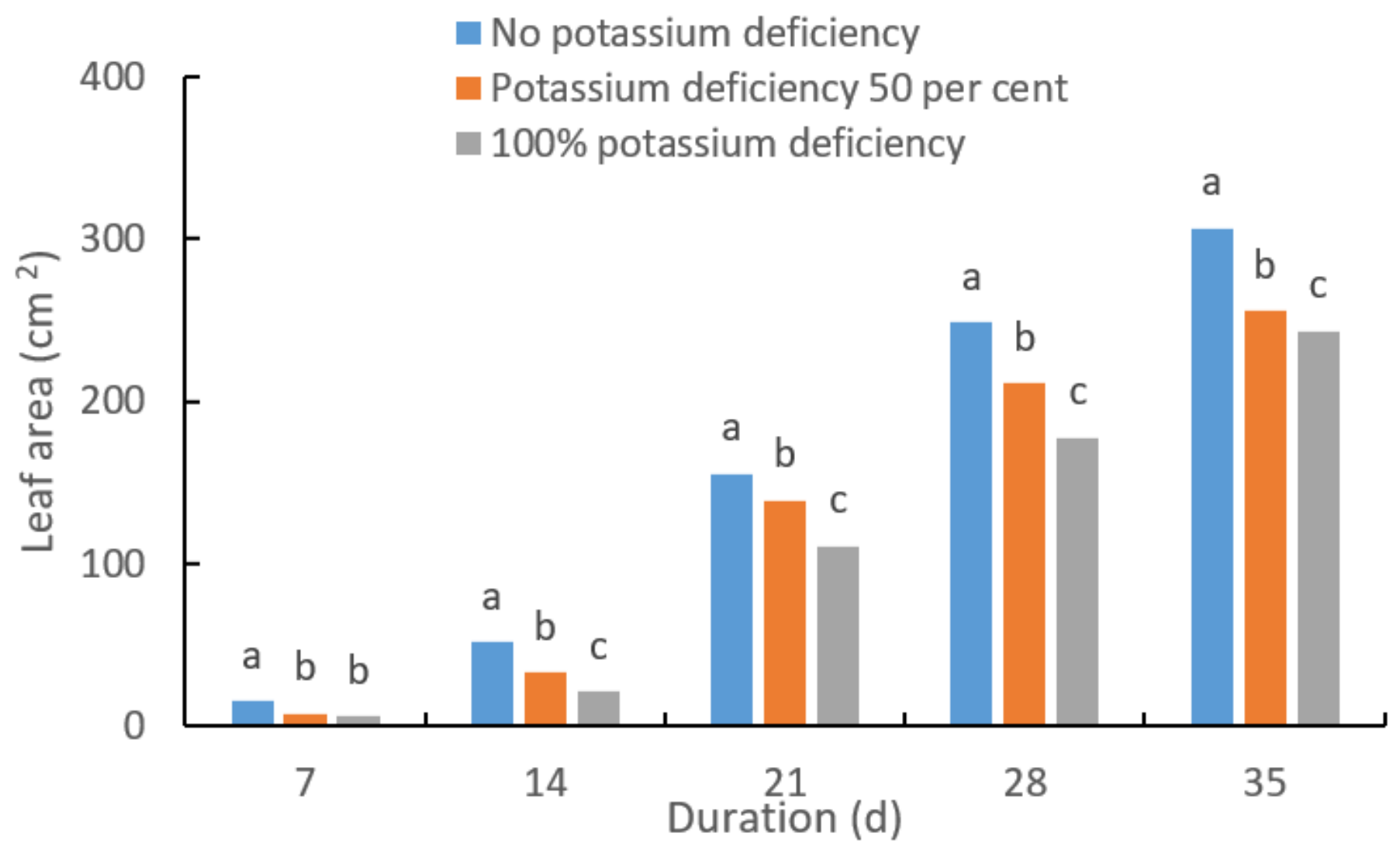

Figure 4

Effect of different potassium treatment on leaf area of Oryza sativa 


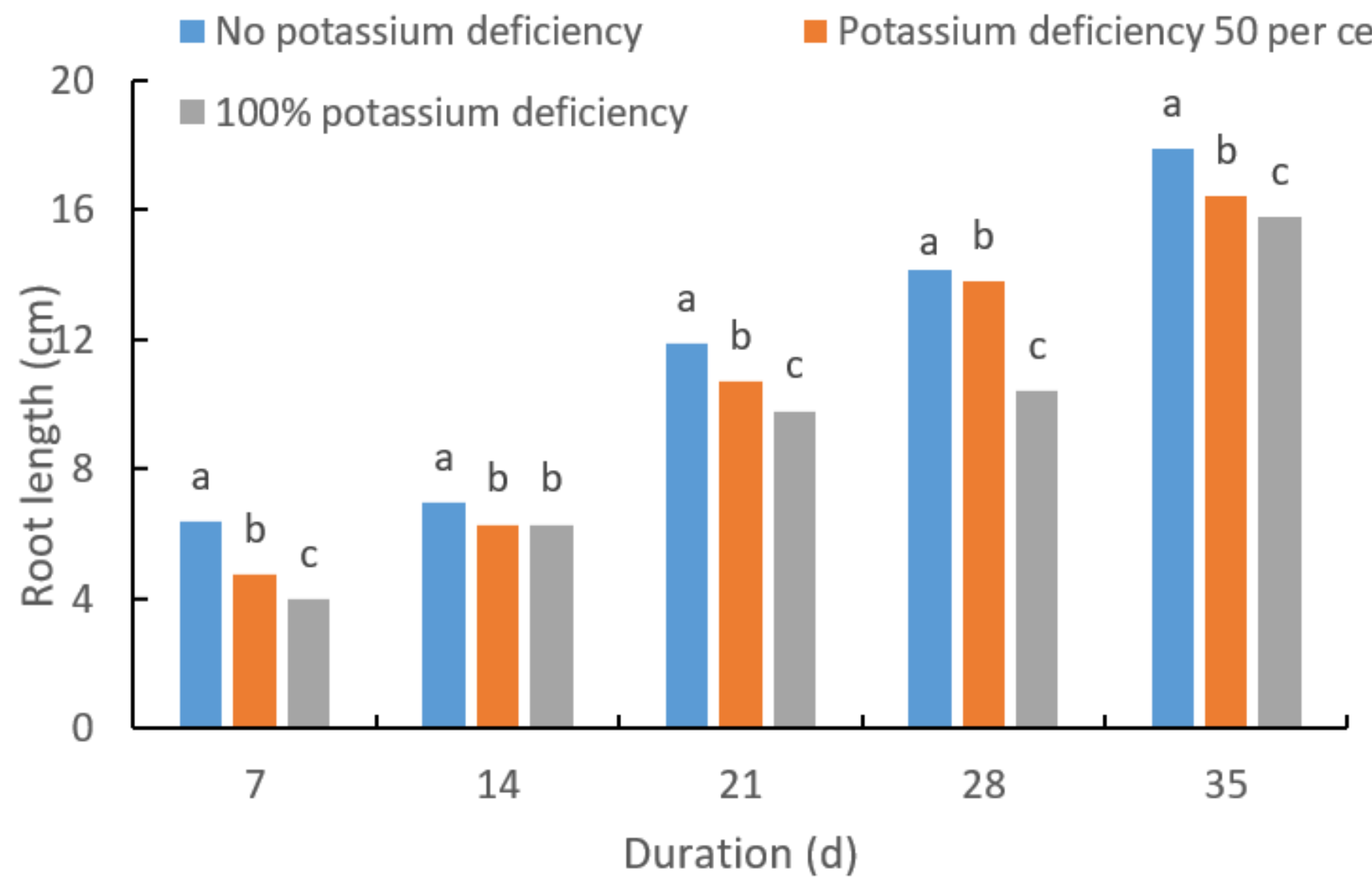

Figure 5

Effects of different potassium treatments on root length of cabbage

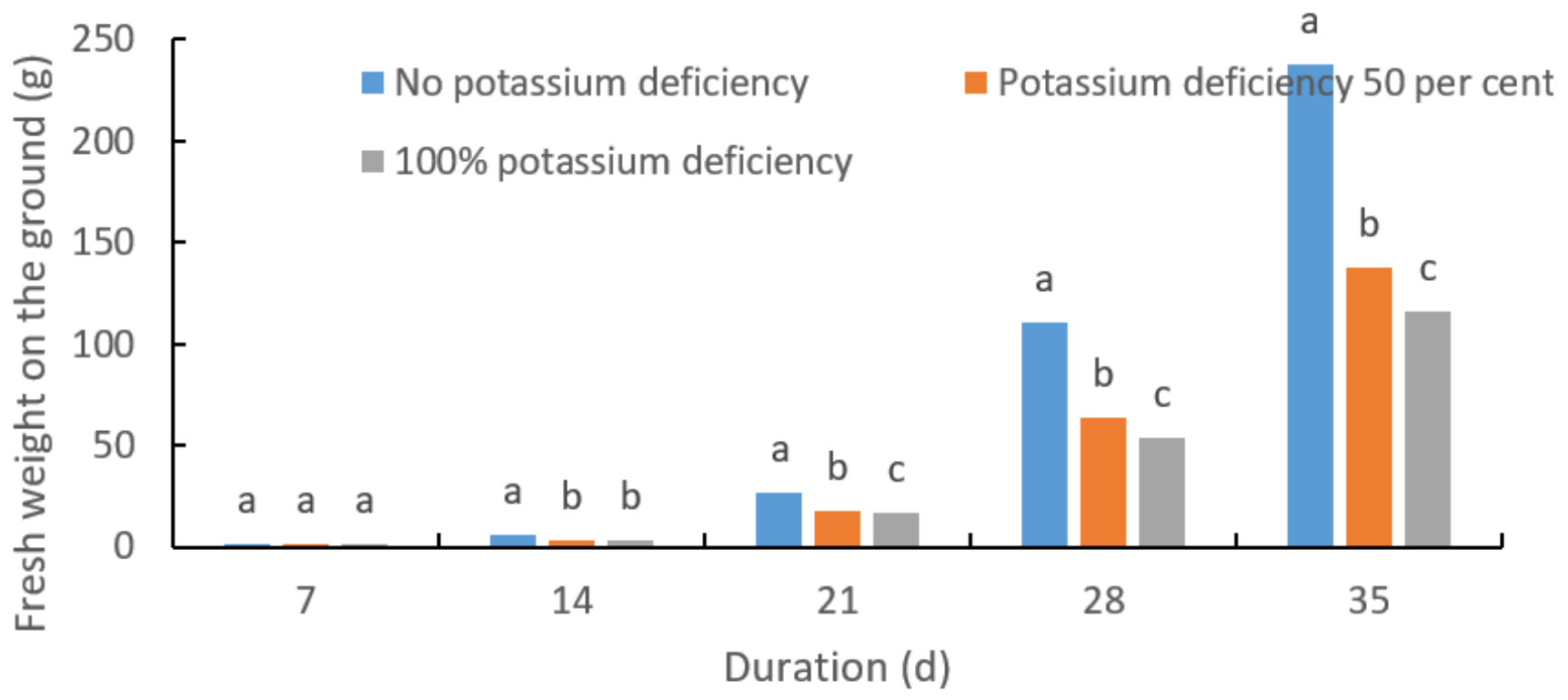

Figure 6 
Effect of different potassium treatment on fresh weight of pakchoi

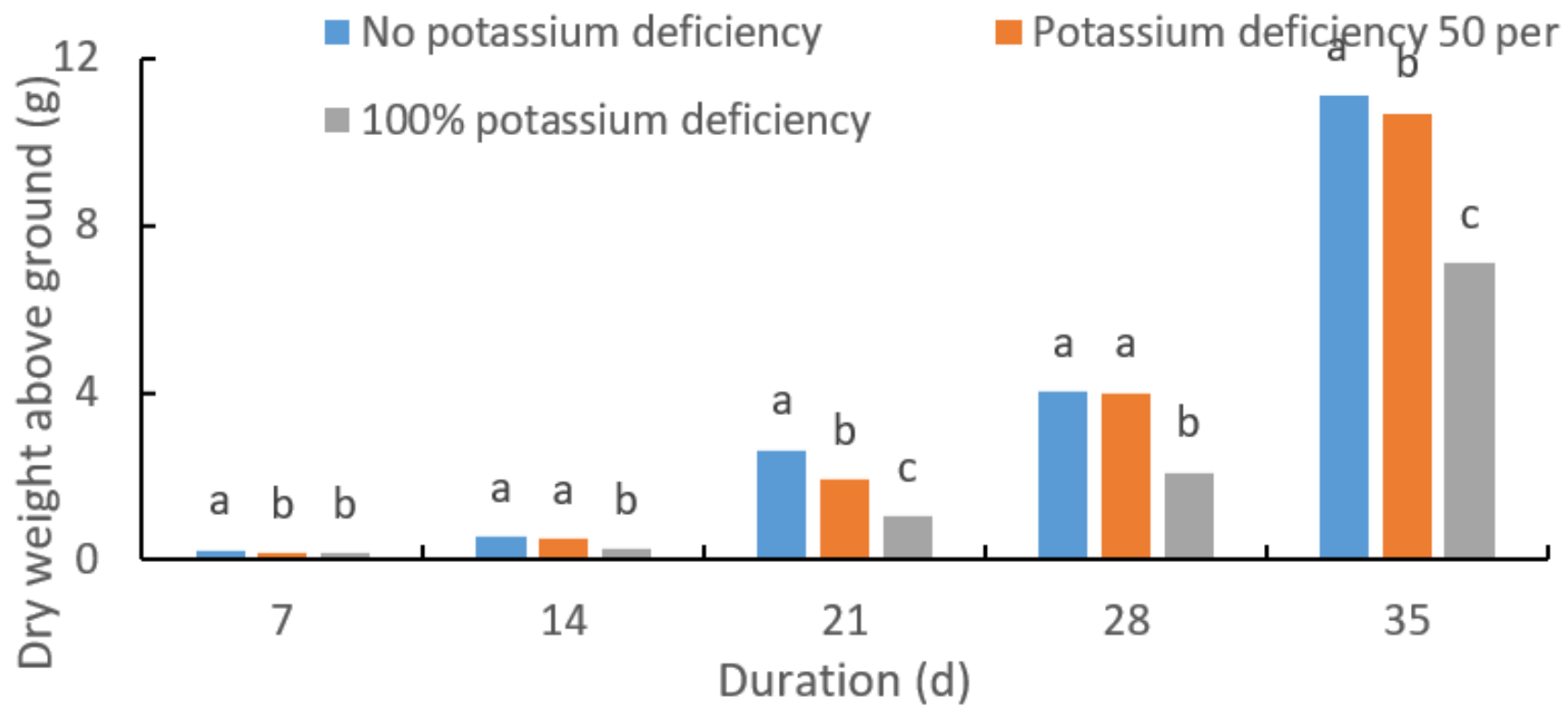

Figure 7

Effect of different potassium treatment on dry weight of upper part of cabbage

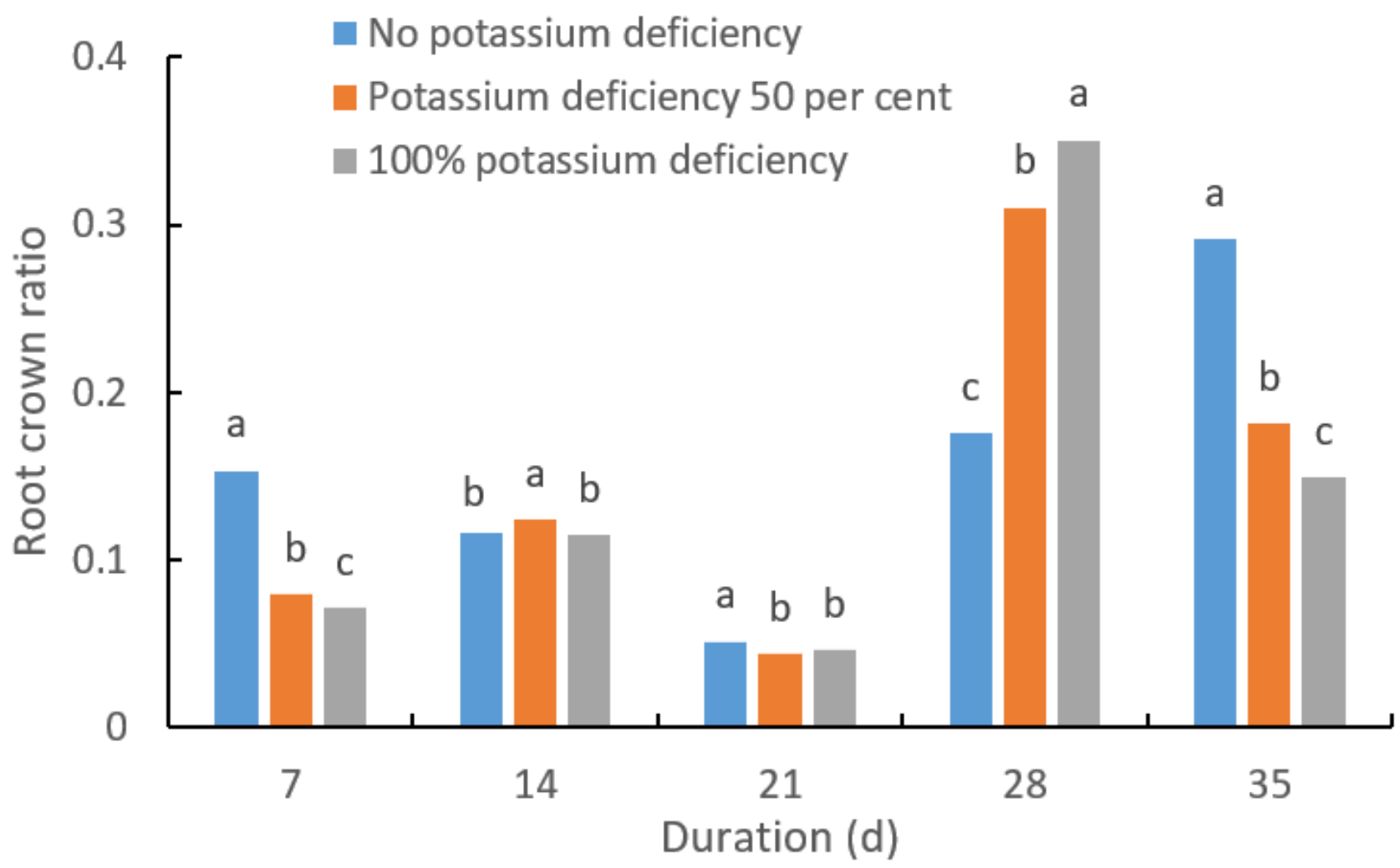

Figure 8

Effect of different potassium treatment on root and crown ratio of pakchoi 


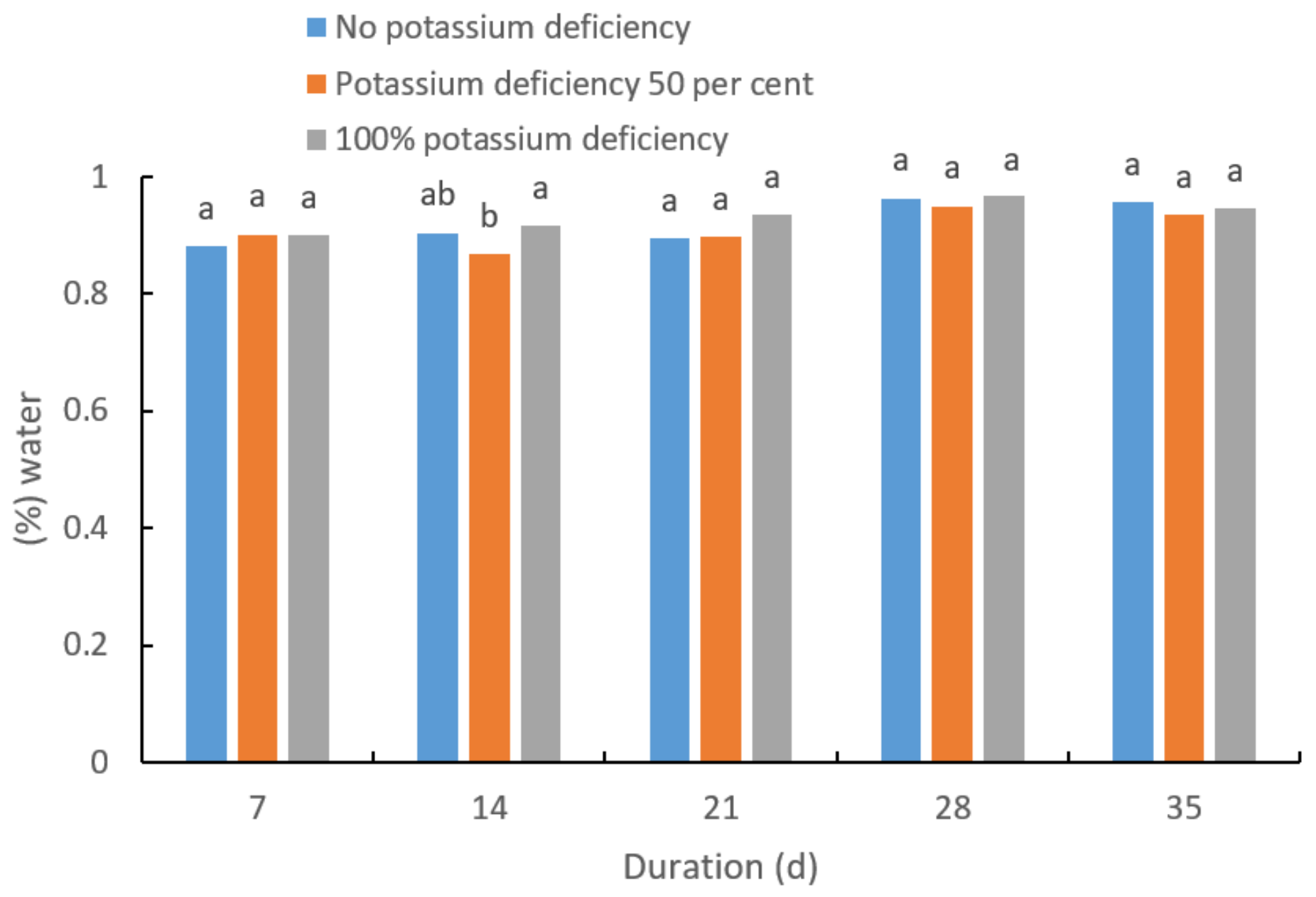

Figure 9

Effect of different potassium treatment on water content of cabbage 